UCRL-JC-131079

PREPRINT

\title{
Laboratory Measurements of Materials in Extreme Conditions: The Use of Uhgh Lnegy Radiation Sources for High Pressure Studies
}

\author{
R. Cauble and B. A. Remington
}

This paper was prepared for submittal to the

Hypervelocity lmpact Symposium

Huntsville, Alabama

November $36-19,1998$

June, 1998






\section{DISCLAIMER}

This document was prepared as an account of work sponsored by an agency of the United States Government. Neither the United States Government nor the University of California nor any of their employees, makes any warranty, express or implied, or assumes any legal liability or responsibility for the accuracy, completeness, or usefulness of any information, apparatus, product, or process

disclosed, or represents that its use would not infringe privately owned rights. Reference herein to any specific commercial product, process, or service by trade name, trademark, manufacturer, or otherwise, does not necessarily constitute or imply its endorsement, recommendation, or favoring by the United States Government or the University of California. The views and opinions of authors expressed herein do not necessarily state or reflect those of the United States Government or the University of California, and shall not be used for advertising or product endorsement purposes. 


\title{
Laboratory Measurements of Materials in Extreme Conditions: \\ The Use of High Energy Radiation Sources for Hight Pressure Studies*
}

R. Cauble and B. A. Remington

Lawrence Livermore National Laboratory, Livermore, CA 94550

\begin{abstract}
High energy lasers can be used to study material conditions that are appropriate fort inertial confinement fusion: that is, materials at high densities, temperatures, and pressures. Pulsed power devices can offer similar opportunities. The National Ignition Facility (NIF) will be a high energy multi-beam laser designed to achieve the thermonuclear ignition of a mm-scale DT-filled target in the laboratory. At the same time, NIF will provide the physics community with a unique tool for the study of high encrgy density matter at states unreachable by any other laboratory technique. Here we describe how these lasers and pulsed power tools can contribute to investigations of high energy density matter in the areas of material properties and equations of state, extend present laboratory shock techniques such as high-speed jets to new regimes, and allow study of extreme conditions found in astrophysical phenomena.
\end{abstract}




\section{Introduction}

The purpose of this article is to introduce and describe the kinds of high energy density experiments that are now being done with high energy radiation sources - lasers and pulsed power devices --on present-day facilities and to indicate where these investigations might proceed with larger facilities either on the drawing board or under construction. Both pulsed power and laser facilities have been funded predominantly for research in inertial confinement fusion (ICF) or, in the case of pulsed power, as high flux, short wavelength $x$-ray sources. The idea that they could be used to investigate specific areas of high energy density physics took some time to germinate. The growth of physics-specific experiments on lasers and pulsed power has been driven largely by the need for high energy density information in the absence of nuclear testing.

Although the use of high energy lasers for studies of, e.g., material properties at extremely high pressure or high-Mach-number hydrodynamic flow, is recent -- less than 10 years, the use of pulsed power machines for such experiments is even newer. Since there is a base of laser experiments on facilities like Nova at Lawrence Livermore National Laboratory (LLNL), the bulk of this article will discuss those experiments. Pulsed power facilities like $Z$ at Sandia National Laboratories (SNL) can and will perform measurements similar to those described below. Extensions of Nova experiments to next generation lasers, like the National Ignition Facility (to be built at LLNL) can be made. The next generation pulsed power machine, designated $\mathrm{X}-1$, may be built at SNL in the next century.

Lasers and pulsed power devices can produce high energy radiation sources but can be seen as somewhat complementary. The most energetic laser for the past 15 years has been Nova[1], although the OMEGA laser[2] at the University of Rochester is now equivalent. Nova is a tenbeam laser that can produce about $100 \mathrm{~kJ}$ of $1-\mu \mathrm{m}$-wavelength laser light. This long wavelength, 
characteristic of a Nd:YaG-doped glass system, turns out to be experimentally less useful than shorter wavelengths (absorption of shorter wavelength light is more efficient and tends to generate less instability at the absorption surface), so the light is most often frequency-tripled to $0.35 \mu \mathrm{m}$. The useable energy of $0.35 \mu \mathrm{m}$ light is $30 \mathrm{~kJ}$. NIF will generate $1.8 \mathrm{MJ}$ of $0.35 \mu \mathrm{m}$ light.[3] Pulsed power machines are more energetic: $Z$ produces $1.8 \mathrm{MJ}$ but the energy is in broadband $\mathrm{x}$-rays.[4] The timescale for high energy lasers like Nova is a few $\mathrm{ns}$, while the timescale for $Z$ is more than $100 \mathrm{~ns}$. Lasers are extraordinarily flexible with respect to the temporal shape of the pulse -- the laser pulseshape can be changed at the oscillator that feeds the amplification system, pulse power facilities must incorporate pulseshaping into the target, design. The spatial scales for laser targets is generally less than $1 \mathrm{~mm}$ while targets on $\mathrm{Z}$ will be several mm. Lasers can focus to very small spots to produce very high temperatures or pressures but often the accuracy of an experiment relies on larger target sizes. In general, Nova can produce more extreme target conditions with better control over how the energy is delivered than $Z . Z$ has the advantage of more energy: $Z$ can deliver its energy over a longer period of time than Nova and do so on multiple targets. The differing drive characteristics of the two facilities means that high energy density experiments, e.g., high pressure equation of state research, will tend to complement each other, but that there will also be a regime where the two will overlap.

\section{Inertial Confinement Fusion, Large Lasers, and Experiments}

Inertial confinement fusion (ICF) has driven the development of large lasers. The goal of ICF is to implode and heat a small capsule containing deuterium and tritium, compressing it with progressively staged shocks to densities and temperatures capable of sustaining thermonuclear ignition. The primary purpose of ICF is the support of two major national activities: maintaining relevant scientific expertise in the absence of nuclear testing and the development of electrical 
energy production from fusion.[5] The driving mechanism will be multi-beam, megajoule-class lasers built with advanced but available technology. The precise timing of the shocks that will implode a DT microsphere requires that the driver be able to deliver the necessary high power on target in a carefully staged manner, thereby controlling the adiabat of the fuel. The National Ignition Facility (NIF)[3] is expected to accomplish this feat in the US; France is planning to build a similar laser called Le Laser Megajoule [6] in France(LMJ). These are billion-dollar facilities. NIF is scheduled to come partially on-line in 2001 and be fully operational in 20032004. The LMJ is scheduled to follow NIF by 6-7 years. A scaled drawing of NIF is shown in Fig 1 .

A typical ignition target will be a thin spherical low-atomic-number (low-Z) shell surrounding a solid DT shell; DT gas will fill the interior. The conditions necessary for ignition of the target require that the fuel be compressed to very high density $\left(10^{3}-10^{4}\right.$ times liquid) and that a central spark region with a temperature of $\sim 10 \mathrm{keV}$ be formed.[7] In order to efficiently compress the fuel, multiple strong shocks (pressures of 1-100 Mbar; $1 \mathrm{Mbar}=100 \mathrm{GPa}$ ) are used so that at ignition the fuel is at enormous pressures but not at high temperature; it will be kept on a low adiabat. Thus the necessary conditions for ignition are an incongruity: a cold, dense fuel shell enclosing a $10 \mathrm{keV}$ hot spot at pressures near $100 \mathrm{Gbar}$ (Fig. 2). This is a most difficult shock physics problem and requires large facilities to accomplish. But the lasers that will used to create these conditions will be unique facilities for producing and diagnosing matter in a range of extreme conditions in the laboratory, high energy-density regimes that have never before been experimentally explored.

These lasers will use either direct or indirect drive to compress the ignition capsule. Both of these methods are used in the high energy-density experiments described in detail in the following sections. Irradiating a target directly with one or more laser beams is called direct drive. At an intensity I the pressure produced in a low-atomic-number material is given by 
$P($ Mbar $) \approx$ few times $\left[I\left(10^{14} \mathrm{~W} / \mathrm{cm}^{2}\right) / \lambda(\mu \mathrm{m})\right]^{2 / 3} \cdot[8] 10^{14} \mathrm{~W} / \mathrm{cm}^{2}$ is not an extraordinary laser intensity, so shocks of 10's of Mbar are possible even with moderate energy lasers, although the intensity may only be achieved over a small spot and for only a very short time duration. Lasers with high energy are needed for most relevant high energy-density experiments. The indirect drive technique uses a high-atomic-number hohlraum into which the laser beams are focused. The laser light is absorbed and re-emitted from the hohlraum wall and then scatters many times within the hohlraum. The resulting $\mathrm{x}$-rays, contained in the hohlraum and characterized by a radiation temperature $T_{r}$, are then used as the driving source. The risetime of $T_{r}$ in the hohlraum follows the risetime of the laser beam itself and can be less than $150 \mathrm{ps}$. The indirect $\mathrm{x}$-ray drive also reflects the shape of the laser profile. The pressure generated in the hohlraum is $\approx 10^{4}$ $T_{r}(\mathrm{keV})^{3.5}$ Mbar.[9] With indirect drive, more beams can be used. Typical hohlraum temperatures are $150-250 \mathrm{eV}$ and shock pressures of $\sim 100 \mathrm{Mbar}$ are common on Nova. NIF hohlraum pressures will be higher, near $500 \mathrm{Mbar}$, and on larger spatial scales.

Pulsed power will be utilized in a manner similar to indirect drive for laser described above. $\mathrm{Z}$, e.g., also uses a hohlraum but whereas the Nova-scale hohlraum is a one-to-a-few mm, the Zscale hohlraum is several $\mathrm{cm}$ in diameter. The source of the radiation is a number of thin metal wires in a cylindrical arrangement through which a large current $(20 \mathrm{MA}$ on $\mathrm{Z})$ is passed. The wires vaporize and $J x B$ forces cause the wires to accelerate toward the axis of the hohlraum. Upon stagnating, the wire plasmas release stored kinetic energy in $\mathrm{X}$-rays. The process is depicted in Fig. 3. The characteristic radiation temperature achieved in the large "primary" hohlraum is $80-120 \mathrm{eV}$. If a cylindrical material is placed on axis so that the wires stagnate on the cylinder, $T_{r}$ in the smaller cylinder can be higher $(\sim 180 \mathrm{eV})$. The risetime for $T_{r}$ in the hohlraum is about $100 \mathrm{~ns}$ with a long precursor and an approximately gaussian profile in time. The precursor can be removed by target design. The arrangement on $\mathrm{Z}$ is to construct two or 
three smaller "secondary" hohlraums on the primary hohlraum wall with experimental packages mounted to the secondary hohlraums.

In addition to producing radiation-driven shock waves, both direct and indirect drive can be used to accelerate a small foil in order to collision with a target, creating a shock in the target that is reminiscent of the more mature high-explosive-driven and gas-gun-accelerated flyer plate experiments. There is a considerable advantage in utilizing this technique: the drive energy is efficiently stored in the flyer and released suddenly as kinetic energy in collision. Indeed, the highest pressure unambiguously recorded in any planar shock in the laboratory - 750 Mbar (75 $\mathrm{TPa}$ - - was created by an gold flyer foil accelerated by $\mathrm{x}$-rays produced in a Nova hohlraum.[10] However, the flyer foil in that experiment was not expected to be "cold," i.e., at a known density, so that no useful EOS data were obtained. In spite of this, it was determined that the spatial dimensions of the target were large enough to use the technique. Early experience with thin directly-driven flyers showed that small spatial nonuniformities in the laser beam would break up the foil rather than accelerating it.[11] However, new research on directly-driven flyers using layered foils show promise.[12] The radiation-driven flyer foil technique will also be tested on the $\mathrm{Z}$ machine.[13]

Kilojoule-class lasers like Nova are being used to investigate fundamentals of high energydensity science. The following sections discuss a few areas of high energy-density physics that are being explored with Nova. These range from Mbar-pressure equation of state measurements to studies of hydrodynamic processes in supernovas. In each case the advantages of performing similar experiments on NIF are described. One very important point: not all high energy-density experiments on NIF will be designed and performed by staff members of the national laboratories. A fraction of the shots will be set aside for use by "outside" users, i.e., university researchers. At this time the fraction of all NIF shots dedicated to outside user is expected to be about $15 \%$. 


\section{Equation of State Experiments at Extreme Conditions}

The experimental and theoretical investigation of equations of state (EOS) of materials at high energy density are of interest not only in ICF, but also in aspects of nuclear explosive design, astrophysics and other related fields. Matter shocked to Mbar pressures is matter that is highly compressed and hot enough to become ionized. The usual theories of condensed matter become difficult to apply as interparticle potentials become more complex. Interparticle interactions exhibit density effects; they are affected by the presence of a large number of nearneighbors. The presence of delocalized electrons and strongly interacting ion cores adds a dynamically screened coulomb component to the mixture of molecular and atomic species. Nevertheless EOS tables that include this regime must be constructed not only to design and interpret ICF target data, but also to study the evolution of large planets and stars. In a regime where the difficulty of the theory is exhibited by the profusion of very different model predictions, EOS data are needed. Most multi-Mbar data have been driven by nuclear explosions in underground tests.[14,15]

Lasers have been known to be capable of producing Mbar shocks for more than a quartercentury[16] and there have been a number of published articles describing strong laser-driven shocks with both direct[17] and indirect[18] drive. But it was not until recently that the first laser-produced multi-Mbar EOS data were published.[19] Laser-driven shock waves have proved difficult to use for Mbar EOS measurements. Several challenges must be overcome when using lasers in such experiments. In order to reduce uncertainties in the data it is necessary to have a large, spatially uniform, steady-state shock front driven for as long as possible. These requires considerable energy, not just a high intensity. The energy required to maintain a given uncertainty in shock speed at pressure $P$ varies as $P^{5 / 2}$.[20] Preventing preheat of the sample is a 
stringent requirement since heating of the sample prior to it being shocked changes the initial sample density in an unknown way. Since laser deposition can produce very high temperatures $(\sim \mathrm{keV})$, sample preheat by $\mathrm{x}$-rays or hot electrons is a major concern for laser-driven experiments. Diagnostics are needed that have few- $\mu \mathrm{m}$ spatial and few $\sim$ ps temporal resolutions. These are formidable challenges to laser-driven EOS measurements but are not insurmountable.[21] Because of the surfeit of energy, larger sample sizes, and lower power (slower) radiation drives, these challenges may be slightly less worrisome for pulse-powerdriven experiments. There is, however, the necessity of working in an electromagnetically rich environment so shielding is a major concern. Finally, a not incidental consideration is the acceptance that radiation-driven measurements will not soon be able to match the experimental precision that researchers have grown accustomed to in sub-Mbar EOS measurements with morc mature techniques. What intense radiation-driven sources offer is the opportunity to explore more extreme regimes that those techniques cannot access.

The laser experiments described below are all Hugoniot measurements (the Hugoniot is the locus of density, pressure, and energy states in a material following passage of a single shock and is a well-defined curve on the EOS surface). Because of the ability of the laser to produce a very fast risetime pulse, a shock can be developed in a target in $\sim 100 \mathrm{ps,} \mathrm{a} \mathrm{time} \mathrm{that} \mathrm{is} 1-10 \%$ of the laser drive time. In contrast, pulsed power machines, with a many-ns risetime and a more gaussian temporal shape, take longer to generate the appropriate shock profile. These characteristics can be readily applied to "shockless" (near-isentropic) high pressure experiments.

EOS experiments have been performed on Nova to investigate materials that are of interest to ICF at relevant Mbar pressures. Hugoniot measurements of polystyrene and beryllium, two candidate materials for the outer shell of NIF ignition capsules, were made using indirect drive. Deuterium, the major constituent of the fuel in fusion capsules was examined using direct drive. 


\section{Indirectly-Driven Hugoniots}

Hugoniot measurements of two low-atomic-number materials have be made on Nova using indirect drive. Indirect drive has some advantages over direct drive. Because $\mathrm{x}$-rays in the hohlraum undergo multiple reflections, the shock generated in a package attached to the hohlraum is spatially uniform; there are no hot spots characteristic of direct irradiation by an unsmoothed laser beam. There is the additional advantage of having a much lower effective drive temperature, reducing the potential for preheating of a sample. The primary disadvantage of using a hohlraum is that the hohlraum fills with plasma and the laser entrance holes close within a few ns; thus shock measurements must be made within this time duration or corrections made for slowing of the shock in the sample.

Polystyrene (CH) and beryllium were the materials examined. Eight beams of the Nova laser were focused into a 3-mm-long by 1.6-mm-diameter gold hohlraum; the EOS package was attached to the side of the hohlraum. See Fig. 4a. Two additional beams irradiated a scandium foil; $x$-rays from this backlighter were used to radiograph the shock moving through the package. A streak camera imaged a slit along the package. The package consisted of two sections, a pusher of bromine-doped $\mathrm{CH}, \mathrm{CH}(\mathrm{Br})$, close to the hohlraum and the EOS sample attached to the $\mathrm{CH}(\mathrm{Br})$. Because $\mathrm{CH}(\mathrm{Br})$ is opaque to the backlighter $\mathrm{x}$-rays, the interface between the two sections is visible on film. Following this interface in time after the shock passes through the $\mathrm{CH}(\mathrm{Br})$ reveals the particle speed behind the shock $U_{p}$. The shock front was also visible as the boundary between the highly transmissive unshocked sample and the less transmissive (higher density) shocked sample. A streak image is shown in Fig. 4b. The shock speed $U_{s}$ was obtained by following this boundary. Putting $U_{s}$ and $U_{p}$ into the Hugoniot relations[22], $\rho=\rho_{o} U_{s} /\left(U_{s}-U_{p}\right)$ and $P=P_{o}+\rho_{o} U_{s} U_{p}$, reveals the shocked pressure and density. Since both $U_{s}$ and $U_{p}$ were measured, the Hugoniot data were absolute data. Data on $\mathrm{CH}$ was obtained from $10 \mathrm{Mbar}$ to 40 
Mbar[23]. Be data[24], shown in Fig. $4 c$, is in the same pressure regime as relative nuclearexplosion-driven experiments.[14,15]

\section{Directly-Driven Hugoniot of Deuterium at the Metal-Insulator Transition}

The first shock in the proposed design for a NIF ignition target will be $900 \mathrm{kbar},[7]$ which lies in a regime where a transition from a diatomic to a monatomic fluid is expected. The transition is complicated by the prediction that pressure ionization is commensurate with molecular dissociation. Various theoretical predictions about how the EOS could be affected by the phase transition[26-32], and even whether the transition was of first order[29,33], had been made since this regime is also important for the description of the atmospheres of Jovian planets and low-mass stars that are $90 \%$ hydrogen (the EOS of all of the hydrogen isotopes are similar;

there is only a scale factor in density between them). Gas gun experiments had been donc[34] but the highest Hugoniot pressure datum was about $200 \mathrm{kbar}$, not high enough to exhibit the predicted effects.

A Nova experiment was designed that utilized a spatially-smoothed laser beam with a 9-ns pulse to drive a strong shock into an aluminum pusher. After transiting the Al the shock propagated into a cryogenic $D_{2}$ sample. Employing side-on radiography, the $A l / D_{2}$ interface and the shock front were imaged in time on a calibrated streak camera. Similar to the indirect drive experiments described above, the motion of the interface provided the particle speed $U_{\mathrm{p}}$, and the motion of the shock front revealed the shock speed $U_{\mathrm{s}}$. See Fig. 5a. Hugoniot data were obtained up to $3.3 \mathrm{Mbar}$ that showed a significantly more compressible EOS (up to 50\%) than had been previously believed.[35] The data, shown in Fig. 5b, include points at that agrees well with the earlier gas gun data. The Nova data closely follow a model that incorporates molecular dissociation in a new way[36]. 
In these experiments a considerable effort was made to minimize preheat. Preheat was monitored by reflecting a probe laser off the rear surface in a Michelson interferometry arrangement.[37] Results showed no detectable preheat. Using a different kind of interferometer, a velocity interferometer, or VISAR, it was shown that the shocked deuterium was highly reflecting, indicating that it had been driven into a metallic state.[38] An optical pyrometer was able to determine the temperature of the shocked deuterium.[39]

\section{The Future of Laser-driven EOS Shock Experiments}

These results were produced using a kilojoule-class laser. A megajoule-class laser will allow directly driven shock experiments up to 50 Mbar over longer drive times (>20 ns) and larger spot sizes. It will be possible to perform absolute EOS measurements with improved accuracy. Scaling of optimized hohlraums from Nova to NIF are expected to allow $\sim 2 \%$ precision EOS measurements at pressures greater than $1 \mathrm{Gbar}$ in an impedance match arrangement. [40]

\section{IIydrodynamic Mix at Migh Energy Density}

Matter in motion at high energy density can be susceptible to hydrodynamic instabilities. In an extreme example, Nova has been used to create high-Mach-number jets $(M=15$ to 30$)$ by using a laser-driven "shaped charge."[41] Instabilities are especially problematic in ICF: mixing of cold fuel into the central hot region can reduce the capsule burn performance or entirely quench the burn.[7] The three most common hydrodynamic instabilities are the accelerationdriven Rayleigh-Taylor (RT) instability, its shock-analog the Richtmyer-Meshkov (RM) instability, and the shear-induced Kelvin-Helmholtz (KH) instability. Mitigating high-energydensity-driven hydrodynamic instabilities is critical in ICF. But they are also observable in astrophysical objects (see Section V). The RT instability can occur at an interface between a low 
density fluid and a high density fluid where the lighter fluid is accelerated into the heavier. In the linear regime, initial perturbations in the interface will grow exponentially in time with a growth rate $\gamma \sim(\mathrm{Ag} / \lambda)^{1 / 2}$, with $A$ being the density-dependent Atwood number, $g$ the acceleration, and $\lambda$ the perturbation wave length. In the nonlinear asymptotic limit, the interface evolves into bubbles of the lighter fluid rising at their terminal velocity and spikes of the heavier fluid falling through the lighter fluid.

High explosives can generate pressures up to 200-300 kbar[42] and gas guns can generate pressures up to a few Mbar in solids, but with modest compression.[43] Large lasers like Nova and NIF can produce extreme accelerations (up to $10^{14}$ earth gravities) and higher pressures. Such lasers can achieve high growth factors, large compressions, and high levels of radiation flow and ionization in arbitrary geometry.

There have been a number of experiments to investigate high energy-density hydrodynamic instabilities using a laser drive. $[9,44-46]$ Because of their flexibility, lasers can provide the variety of conditions necessary to probe the evolution of RM and RT instabilities. One important example is the experimental verification of the differences between two-dimensional (2D) and three-dimensional (3D) RT-induced perturbation growth[30] (this result will be important in the following section). A perturbation was pre-imposed on one side of $\mathrm{a} \mathrm{CH}(\mathrm{Br})$ foil. This foil was mounted across an opening on a laser hohlraum that then ablatively accelerated the foil. The experimental arrangement is similar to that in Fig. $4 a$, except that the backlighter and detector are positioned so that radiographic images obtained are face-on (through the foil) rather than side-on. Perturbations at the ablation front grow due to the RT instability. The three perturbations studied all had the same magnitude wavevector, $\mathrm{k}=\left(\mathrm{k}_{\mathrm{x}}{ }^{2}+\mathrm{k}_{\mathrm{y}}{ }^{2}\right)^{1 / 2}$, where $k=2 \pi / \lambda$, and amplitude, differing only in their shape. The labels are: 3D square $k_{x}=k_{y}, 3 D$ stretched $k_{x}=3 k_{y}$, and 2D ripple $k=k_{x}=k_{2 D}$. Time-resolved, face-on radiographs are shown in Fig. 6a. Dark regions correspond to spikes, bright regions to bubbles. The growth versus time of the fundamental mode Fourier 
amplitudes of the perturbations are shown in Fig. 6b. In the linear regime, all three modes grow at the same rate. This is expected from linear theory since they all have the same magnitude wavevector. However, in the nonlinear regime, the 3D square mode grows the largest, the 2D ripple grows the least, and the 3D stretched perturbation falls in between. This result is not unexpected. At the bubble tip, the ratio of drag over buoyancy is smallest for the square mode; basically, the square mode bubble is more streamlined.[48] This shape has the highest terminal bubble velocity and grows the fastest. The energy of Nova is sufficient to just begin to see these effects. The energy available on NIF will allow 3D experiments to be pushed well into the nonlinear regime. Furthermore we will also be able to perform experiments on NIF with fully multimode 3D foils, not possible on Nova. It is important to drive instability experiments longer in order to observe the growth with better accuracy. The longer drives available on $\mathrm{Z}$ make $\mathrm{Z}$ favorable for hydrodynamic instability experiments.

Three-dimensional effects in $\mathrm{RT}$ development can also be seen in convergent geometry. This is evident in preliminary Nova experiments in which a 3D square $\left(\mathrm{k}_{\mathrm{x}}=\mathrm{k}_{\mathrm{y}}\right)$ mode perturbation was inscribed on the outer surface of a doped-plastic hemisphere and, similar to the planar RT experiments described above, the hemisphere was mounted on the wall of a hohlraum, facing inwards. [49] As in the planar case, the perturbations grow as the plastic target is accelerated. But since the hemisphere converges as it is driven, the perturbation wavelength $\lambda$ decreases as the shell accelerates. Recalling that the RT growth rate $\sim \lambda^{-1 / 2}, \mathrm{RT}$ growth was expected to be larger in the linear regime in the convergent case and to saturate sooner. This is indeed what was observed. Although the convergence ratio $R_{o} / R$, where $R_{o}$ is the initial hemisphere radius and $R$ the final radius, was only about two, RT growth entered the nonlinear regime. Had the foil been planar, the growth would still be in the linear regime at the times observed. Megajoule-class lasers will be required to drive these 3D experiments well into the nonlinear regime especially, as in the case of planar $\mathrm{RT}$, with multimode targets. The ability to perform fully $3 \mathrm{D}$ experiments to 
study high energy-density hydrodynamics finds application not only in ICF but also in astrophysics, as shown in the following section.

Hydrodynamics at high pressure can also be studied in the solid state. Careful tailoring of the drive pulse in a hohlraum can result in Mbar-pressure shock waves in a metallic targets without melting the target. Recent experiments have shown that $\mathrm{RT}$ growth in solid $\mathrm{Cu}$ is reduced because of strength inherent in the solid.[50] Those experiments also showed that the RT growth reduction could not be explained by extrapolating to Mbar pressures material strength models known to be correct only in the kbar regime. Thus, without these data, Mbar-pressure material strength in $\mathrm{Cu}$ would have been underestimated by a factor of 30 . As a complement to these integral experiments, high pressure shock processes in solids can be investigated in situ by timeresolved $x$-ray diffraction. [51] As a condensed matter sample deforms under shock loading, the lattice spacing changes and thus the Bragg diffraction angle. Monitoring the diffraction angle as a shock propagates through the sample allows the structure of the shock front to be examined. In situ diffraction of a solid shock-compressed to $300 \mathrm{kbar}$ has recently been reported.[52] NIF will be able to drive still larger pressures as well as provide additional and higher flux backlighters for more careful measurements.

\section{Laboratory Experiments and Supernovas}

The application of high energy density experiments to astrophysics was discussed in Section III, where Nova-driven equation of state measurements were found to be important for some low-mass stars. It turns out that laboratory experiments can be directed toward the understanding of far more energetic astrophysical objects. Because of enomous gravitational and nuclear forces, many astrophysical objects are indeed high energy-density phenomena. Exploding stars can create high speed hydrodynamical jets, not dissimilar to those already described.[53] 
Supernovas in particular exhibit a range of high energy-density hydrodynamical features that can be scaled to experiments on megajoule-class lasers. Here we describe two experiments developed to investigate supernova phenomena: (1) hydrodynamic-instability-driven core mixing during the SN1987A explosion and (2) strong shock formation in the SN1987A remnant evolution. These experiments are described in detail in Ref. 54.

\section{SN1987A Mix Experiments}

Gravitational stratification leads to a model of a supernova (SN) progenitor star that is a series of concentric shells with the lightest element, hydrogen, forming the outer envelope, helium just below the H layer, and so on to an iron core. A SN explosion occurs when the Fe core undergoes catastrophic gravitational collapse; a strong radial shock is then driven outward through the star. At the H/He boundary, the post-shock density, temperature, and pressure are about $2.3 \mathrm{~g} / \mathrm{cm}^{3}, 6 \mathrm{keV}$, and $75 \mathrm{Gbar}$. Much effort has been invested in developing models to understand the underlying processes of SNs. Virtually all of these efforts have focused on onedimensional (1D) stellar evolution models, treating multidimensional hydrodynamics effects with prescriptions from mixing length theory. Observations of SN1987A highlighted two distinct defects with these models.[55]

One means of evaluating SN models is through the light curve, the total luminosity of the SN versus time. The SN luminosity decreases sharply immediately after the explosion as ejected gas hydrodynamically cools due to expansion. Subsequently the luminosity exhibits a broad peak as the heat wave due to radioactive decay of the core diffuses out of the star. In SN1987A, however, the core became visible much earlier than 1D diffusion models predicted.[56] The reason was traced to unexpected mixing of the core with the stellar atmosphere sitting on top of the core. Concentrating on just the $\mathrm{H}$ and He layers, the boundary between the shells is RichtmyerMeshkov unstable as the shock crosses the interface. Further, after shock passage both layers are 
accelerated, but the heavier He decelerates into lighter $\mathrm{H}$; the interface becomes unstable to Rayleigh-Taylor (RT) growth so that initial density perturbations continue to grow. The observations from SN1987A provided compelling evidence of the need for two-dimensional (2D) stellar models.[57] Multi-dimensional SN simulations later showed that RT-induced mix led to earlier emergence of core radiation compared to 10 models. [58]

Even after $2 \mathrm{D}$ SN models were developed, there remained a difficulty. These codes predicted peak core velocities of $<2000 \mathrm{~km} / \mathrm{s}$ for SN1987A. However, Doppler broadening showed core velocities that were much higher, $>3000 \mathrm{~km} / \mathrm{s}$. [59] The solution of this problem can be seen in the results of laser mix experiments described in the preceding section. There it was noted that strongly driven RT growth was observed to be larger in target foils where the perturbations resembled squares $(3 \mathrm{D})$ compared to $(2 \mathrm{D})$ ripple-like perturbations. [47] This was an indication that even $2 \mathrm{D}$ modeling was insufficient and $3 \mathrm{D}$ codes must be developed and used.

Multi-dimensional SN simulation codes have thus evolved within the last ten years. It is reasonable to ask about the accuracy of these codes. How can they be benchmarked in a meaningful way?

Noting, somewhat remarkably, that we can scale supernova hydrodynamics to the millimeter scales of laser-driven experiments, attempts to simulate SN hydrodynamics have been performed on the Nova laser using a hohlraum $\mathrm{x}$-ray drive with $\mathrm{Cu}$ and $\mathrm{CH}_{2}$ as surrogates for $\mathrm{He}$ and $\mathrm{H}$ respectively. [54] The indirectly driven shock tube arrangement (as in Fig. 4a) was utilized. The flexibility inherent in laser energy delivery made a scaled representation of the correct shockplus-deceleration drive possible. In the experiment, side-on radiographs of the interface show how far and how fast bubbles and spikes form due to RM followed by RT instability. These images are compared to code predictions. Employing a single mode 2D surface (see Fig. 6), gross features of the experiment are reproduced by the simulations.[60] However there is 
insufficient drive energy available with Nova to allow these experiments to be done in a multimode $3 \mathrm{D}$ configuration or experiments in (the correct) exploding geometry. However, NIF will have sufficient energy to drive more realistic experiments.

\section{SN1987A Remnant Collision Experiments}

SN1987A is now evolving into the early remnant stage; a 1994 optical image is shown in Fig. 7. The expanding SN ejecta is the central bright spot, surrounded by an assembly of nebular rings, the origin of which is a mystery. Gaseous $S N$ ejecta, moving at $\sim 10^{3}-10^{4} \mathrm{~km} / \mathrm{s}$, will collide with the inner circumstellar ring in a few years; observations of the collision may shed light on the nature of the rings. [57] However, using this collision to probe the nature of the rings depends on correctly interpreting observations of a complex, energetic, hydrodynamically unstable collision between two interstellar plasmas. Simulations are being performed now to predict the outcome of the collision.

When the two plasmas collide, shocks will be launched forward into the ring and backward into the SN ejecta. Cooling of the strongly shocked plasma by radiation causes the compressed ejecta to collapse to an even higher density leading to strong $\mathrm{RT}$ growth at the contact discontinuity. [61] Qualitatively different mixing evolves depending on the density profile of the ejecta and the initial evolution of the contact discontinuity. Large differences also result from inclusion of radiation transfer in the simulations; shock cooling is a crucial part of this process. It is therefore beneficial to test various models experimentally prior to the collision in order to assess how astrophysics codes handle the complex radiative plasma dynamics.

An experiment has been designed as a proxy to the stellar collision. [54] In the experiment, $\mathrm{CH}(\mathrm{Br})$ is used to provide a surrogate for the $\mathrm{SN}$ ejecta; $\mathrm{SiO}_{2}$ aerogel foam represents ambient plasma and a solid $\mathrm{CH}$ foil corresponds to the ring plasma. Attached to a Nova hohlraum, $\mathrm{x}$-rays drive a $50 \mathrm{Mbar}$ shock into the $\mathrm{CH}(\mathrm{Br})$, ejecting plasma into a gap between the $\mathrm{CH}(\mathrm{Br})$ and the 
foam. The ejecta stagnates in the foam driving shocks forward into the foam and backward into the ejecta. Side -on x-ray backlighting reveals the positions of the shocks and the relative shock densities. Experiments on Nova have successfully produced and imaged both shocks. However, the shocks are not strong enough so that cooling radiation is a factor in the Nova experiment. Further, longer X-ray drives are needed to evince the expected RT development in the plasma. NIF will provide the drive energy to overcome both of these difficulties.

\section{Summary}

A primary mission of megajoule-class lasers such as NIF will be to compress, inertially confine, and initiate nuclear fusion in DT capsules. This approach to ICF ignition requires a flexible, high-energy facility capable of producing conditions of matter unattainable by other laboratory methods while simultaneously allowing experimental investigation of shocked, high

energy-density matter. Several examples of high energy-density experimental investigations have been described that have already been initiated on present-day kilojoule-class lasers and how those studies will be extended on NIF. The examples described included material properties at Mbar pressures, production of high-Mach-number jets, and the growth and development of hydrodynamic instabilities in planar and convergent geometries that reveal the limitations of two-dimensional models. High energy lasers can even make contributions to the study of shocks in astrophysical phenomena, in particular supernovas. Further details on these experiments, and on many others, can be found in Ref. 62, which is an explorable website. It is expected that pulsed power devices, such as Z and $\mathrm{X}-1$, if it is built, will enhance the repertoire of high energy density experiments. Experiments at NIF will be performed not only by the staff at US national laboratories, but also in collaboration with and by members of the physics academic community.[63] 


\section{Ackmowledgments}

*This work was performed under the auspices of the U.S. Department of Energy by the Lawrence Livermore National Laboratory under contract No. W-7405-ENG-48 


\section{References}

1. E.M. Campbell, Laser Part. Beams 9, 209 (1991).

2. J. M. Soures et al., Phys. Plasmas 3, 2108 (1996).

3. J. A. Paisner, E.M. Campbell, and W.J. Hogan, Fusion Technology 26, 755 (1994).

4. M. K. Matzen, Phys. Plasmas 4, 1519 (1997).

5. E. M. Campbell, N. C. Holmes, S. B. Libby, R. A. Remington, and E. Teller, Laser Part. Beams 15, 607 (1997).

6. M. André, "Conceptual Design of the French LMJ Laser," First SPIE International Conference on Solid State Lasers for Application to ICF, Monterey, (1995).

7. John Lindl, Phys. Plasmas 2, 3933 (1995).

8. Th. Löwer and R. Sigel, Contrib. Plasma. Phys. 33, 355 (1993).

9. B. A. Remington et al., Phys. Plasmas 2, 241 (1995).

10. R. Cauble et al., Phys. Rev. Lett. 70, 2102 (1993).

11. R. Caubleet al., Phys. Rev. Lett. 74, 3816 (1995).

12. K. A. Tanaka, proceedings of the Worskhop on Equation of State of Hydrogen at Iligh Energy Density, Livermore (1998).

13. J. R. Asay, private communication (1998).

14. C. E. Ragan III, Phys. Rev. A 29, 1391 (1984). 
15. W. J. Nellis, J. A. Moriarity, A. C. Mitchell, and N. C. Holmes, J. Appl. Physics 82,2225 (1997).

16. C. G. M. van Kessel and R. Sigel, Phys. Rev. Lett. 33, 1020 (1974); L. R. Veeser and S. C. Solem, Phys. Rev. Lett. 40, 1391 (1978); R. J. Trainor, J. W. Shaner, J. M. Auerbach, and N. C. Holmes, Phys. Rev. Lett. 42, 1154 (1979).

17. S. P. Obenschain et al., Phys. Rev. Lett. 50, 44 (1983); F. Cottet, J. P. Romain, R. Fabbro, and B. Faral, Phys. Rev. Lett. 52, 1884 (1984); R. Fabbro et al., Laser Part. Beams 413 (1986); K. A. Tanaka et al., in Shock Waves, ed. K. Takayama (Springer-Verlag, Berlin, 1992), p. 863.

18. Th. Löwer et al., Phys. Rev. Lett. 72, 3186 (1994).

19. A. Ng, D. Parfeniuk, and L. D. Da Silva, Phys. Rev. Lett. 54, 2604 (1985); M. Koenig et al., Phys. Rev. Lett., 74, 2260 (1995); S. Fu, Y. Gu, J. Wu, and S. Wang, Phys. Plasmas 2, 3461 (1995); A. Benuzzi et al., Phys. Rev. E 54, 2162 (1996); M. Evans et al., Lasers Part. Beams 14, $113(1996)$.

20. N. C. Holmes, private communication.

21. Y. M. Gupta and S. M. Sharma, Science 277, 909 (1997).

22. Y. B. Zel'dovich and Y. P. Raizer, Physics of Shock Waves and High-Temperature Hydrodynamic Phenomena (Academic Press, New York, 1966).

23. R. Cauble et al,, Phys. Plasmas 4, 1857 (1997).

24. R. Cauble et al, Phys. Rev. Lett. 80, 1248 (1998). 
25. S. P. Lyon and J. D. Johnson, "SESAME: The Los Alamos National Laboratory Equation of State Database," Los Alamos National Laboratory report LA-UR-92-3407 (1992).

26. G. I. Kerley, "A Theoretical Equation of State for Deuterium," Los Alamos Laboratory Report LA-4776 (1972); G. Kerley, J. Chem. Phys. 73,460 (1980).

27. M. Ross, F. H. Ree, and D. A. Young, J. Chem. Phys. 79, 1487 (1983).

28. D. Saumon and G. Chabrier, Phys. Rev A 44, 5122 (1991); 46, 2084 (1992).

29. W. R. Magro, D. M. Ceperley, C. Pierleoni, and B. Bernu, Phys. Rev. Lett. 76, 1240 (1996);

B. Militzer, W. Magro, and D. Ceperley, Proceedings of the International Conference on Strongly Coupled Coulomb Systems, Boston (1997).

31. T. J. Lenosky, J. D. Kress, and L. A. Collins, Phys. Rev. B $\$ 6,5164$ (1997).

32. F. J. Rogers, Astrophys. J. 310, 723 (1986); F. J. Rogers, F. J. Swenson, and C. A. Iglesias, Astrophys. J. 456, 902 (1996).

33. D. Saumon and G. Chabrier, Phys. Rev. Lett. 62, 2397 (1989).

34. W. J. Nellis et al., J. Chem. Phys. 79, 1480 (1983); N. C. Holmes, M. Ross, and W. J. Nellis, Phys. Rev. B 52, 15835 (1995).

33. L. B. Da Silva et al., Phys. Rev. Lett. 78,483 (1997); G. W. Collins et al., submitted to Science (1998).

35. M. Ross, Phys. Rev. B, in press (1998).

37. K. S. Budil et al. "Characterization of Laser-Driven Shock Waves using Interferometry," to appear in Inertial Confinement Fusion Quarterly Report, LLNL report (1997). 
38. P. M. Celliers et al, submitted to Science (1998).

39. G. W. Collins et al., submitted to Phys. Rev. Lett. (1998).

40. N. Landen, private communication (1997).

41. P. L. Miller et al., "Shock-hydrodynamic Experiments on Nova," Proceedings of the 20th International Symposium on Shock Waves," Pasadena (1995).

42. R.F. Benjamin and J.N. Fritz, Phys. Fluids 30, 331 (1987).

43. N.C. Holmes et al., Appl. Phys. Lett 45, 626(1984).

44. K. Budil et al., Phys. Rev. Lett. 76, 4536 (1996); G. Dimonte et al., Phys. Rev. Lett. 74, 4855 (1995); T.A. Peyser et al, Phys. Rev. Lett. 75, 2332 (1995).

45. W. Hsing et al., Phys. Rev. Lett. 78, 3876 1997); Phys. Plasmas 4, 1832 (1997).

46. K. Nishihara and H. Sakagama, Phys. Rev. Lett. 65, 432 (1990).

47. M. Marinak et al., Phys. Rev.Lett. 75, 3677 (1995);M. Marinak et al., Phys. Plasmas 5, 1125 (1998).

48 J. Hecht et al., Laser and Part. Beams 13, 423 (1995).

49. S.G. Glendinning et al., in Proceedings of the 6th International Workshop on the Physics of Compressible Turbulent Mixing, Marseille, in press (1997).

50. D.H. Kalantar, in the proceedings of the 6th International Workshop on the Physics of Compressible Turbulent Mixing, Marseille (1997).

51. J. S. Wark et al., J. Appl. Phys. 68,4531 (1990). 
52. D.H. Kalantar, et al., "Transient X-ray Diffraction Used to Diagnose High Pressure Shocked Solids," Annual Meeting of the American Cyrstallographyic Association, St. Louis (1997).

53. S. Heathcote et al., Astron. J. 112, 1141 (1996).

54. B. A. Remington et al., Phys. Plasmas 4, 1994 (1997).

55. I. Hachisu, T. Matsuda, K. Nomoto, T. Shigeyama, Astron. Astrophys. Suppl. 104, 341

(1994); M. Herant and S.E. Woosley, Astrophys. J. 425, 814 (1994).

56. T. Shigeyama and K. Nomoto, Astrophys. J. 360, 242 (1990).

57. R. McCray, Ann. Rev. Astron. Astrophys. 31, 175 (1993).

58. D. Arnett, Astrophys. J. 427, 932 (1994).

59. B. Fryxell, W. M?ller, and D. Arnett, Astrophys. J. 367, 619 (1991).

60. J. Kane et al., Astrophys.J. 478, L75 (1997).

61. R.A. Chevalier and J.M. Blondin, Astrophys. J. 444, 312 (1995).

62. R. W. Lee, "Science on High Energy Lasers from Today to the NIF," at http://www.llnl.gov/science_on_lasers/ucrl119170.html. Copies of the report can be obtained by contacting $R$. W. Lee at LLNL.

63. See the report "Facility Use Plan for the National Ignition Facility," eds. A. Hauer, R. Kauffman, A. Satsangi, T. Haill, R. Cauble, and T. Saito, available from US Department of Energy, Office of the NIF, 1000 Independence Avenue, S.W. Washington, DC 20585. 


\section{Figure Captions}

Fig. 1 A scaled drawing of the National Ignition Facility being constructed at Lawrence Livermore National Laboratory.

Fig. 2. Schematic compression of an ICF ignition target showing heating of the ablator and corresponding rocket-like implosion of fuel. The resulting ignition configuration is also depicted. Only precisely staged strong shocks from a carefully shaped drive can lead to ignition.

Fig. 3 a) Photograph of a wire array "load" for the Z pulsed power machine at Sandia National Laboratories. A high current passed through the wires and magnetic forces cause the vaporized wires to pinch on the cylindrical axis, as indicated in (b). Radiation form the pinch can be used to drive high energy density experiments.

Fig. 4. a) Target for the Nova measurement of the Hugoniot of low-atomic-number solids by indirect laser drive employing a gold hohlraum. The experimental package is mounted to the side of the hohlraum. The measurements were made with streak radiography using $\mathrm{x}$-rays from a laser-heated backlighter; an image is shown in (b). c) Absolute beryllium Hugoniot data[24] compared with SESAME EOS table Hugoniot[25] and nuclear impedance match data[14,15].

Fig 5. a) Cryogenic cell for the Nova measurement of the Hugoniot of deuterium by direct laser drive, including interferometry to verify preheat. b) Absolute $D_{2}$ Hugoniot data[35] along with gas gun data (triangles)[34] compared with theoretical results: SESAME (solid)[25,26]; molecular dynamics (dot-dash)[31]; Monte Carlo (dash-double-dot)[29]; ACTEX (chain dash)[32]; Saumon and Chabrier (dashes)[28]; and linear mixing (dots)[35].

Fig. 6. a) Face-on radiographs of three different planar Rayleigh-Taylor targets driven indirectly by Nova lasers. The experiment used a hohlraum as in Fig. 4a, except that the backlighter was 
positioned above the hohlraum and the recording device, an $\mathrm{x}$-ray framing camera, was

positioned below. The three shapes are $3 D$ square $k_{x}=k_{y}, 3 D$ stretched $k_{x}=3 k_{y}$, and $2 D$ ripple $\mathrm{k}=\mathrm{k}_{\mathrm{X}}=\mathrm{k}_{2 \mathrm{D}}$. b) Plot of perturbation Fourier amplitude vs. time for the three shapes showing that the 3D square mode grows fastest and the 2D mode grows slowest.[47]

Fig. 7. An image of SN1987A obtained by the Hubble Telescope in Feb. 1994. The expanding supernova ejecta is the central dot. The rings are planetary nebulae of uncertain origin will be impacted by ejecta from the supernova in a few years. 


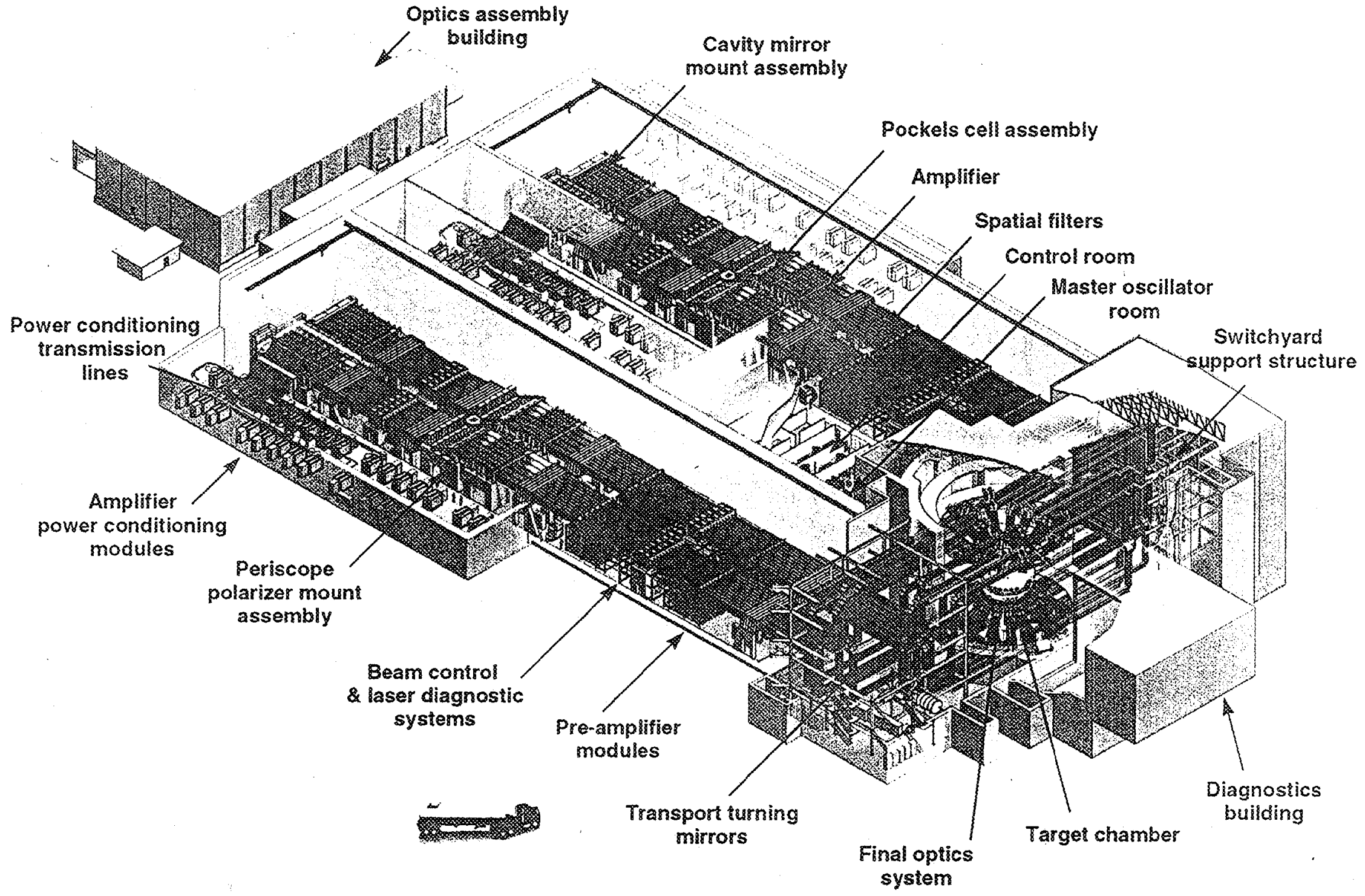




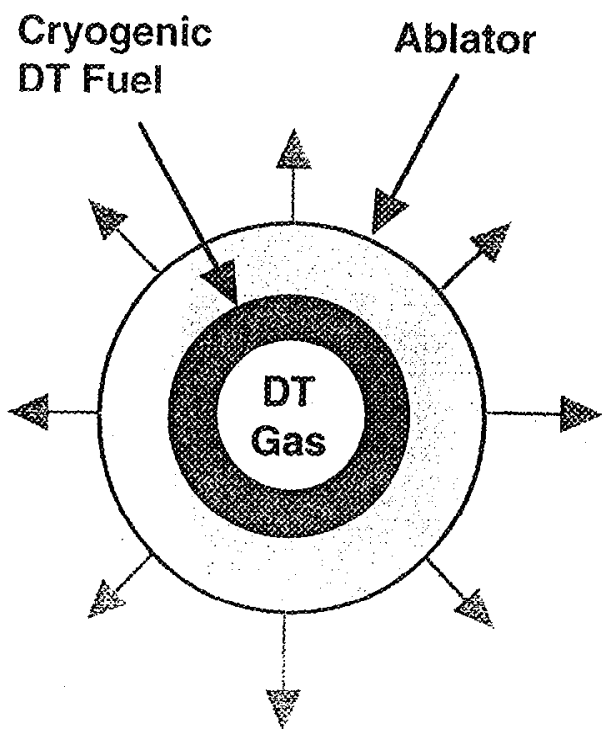

\section{Degenerate Fuel}

$200-1000 \mathrm{~g} / \mathrm{cm}^{3}$








\section{Wire Array}

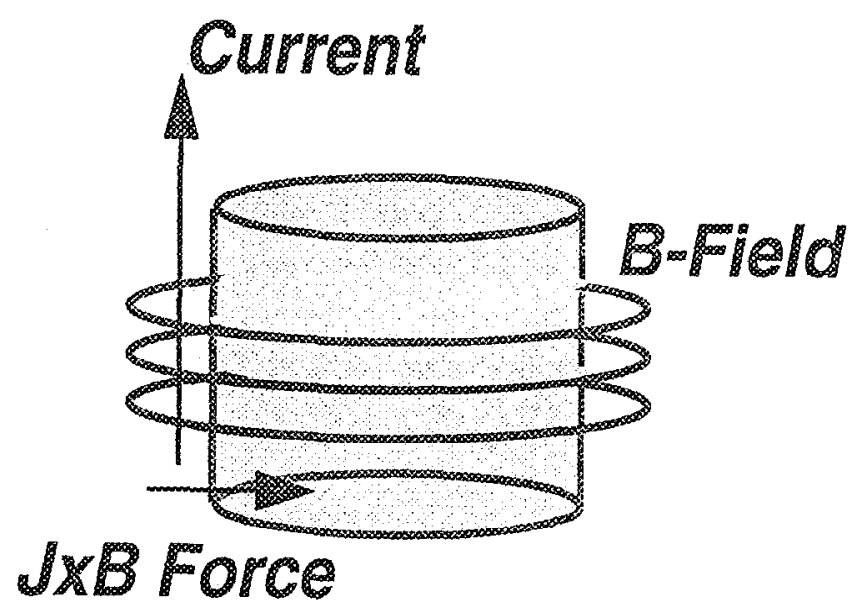

Cauble Fig 3 


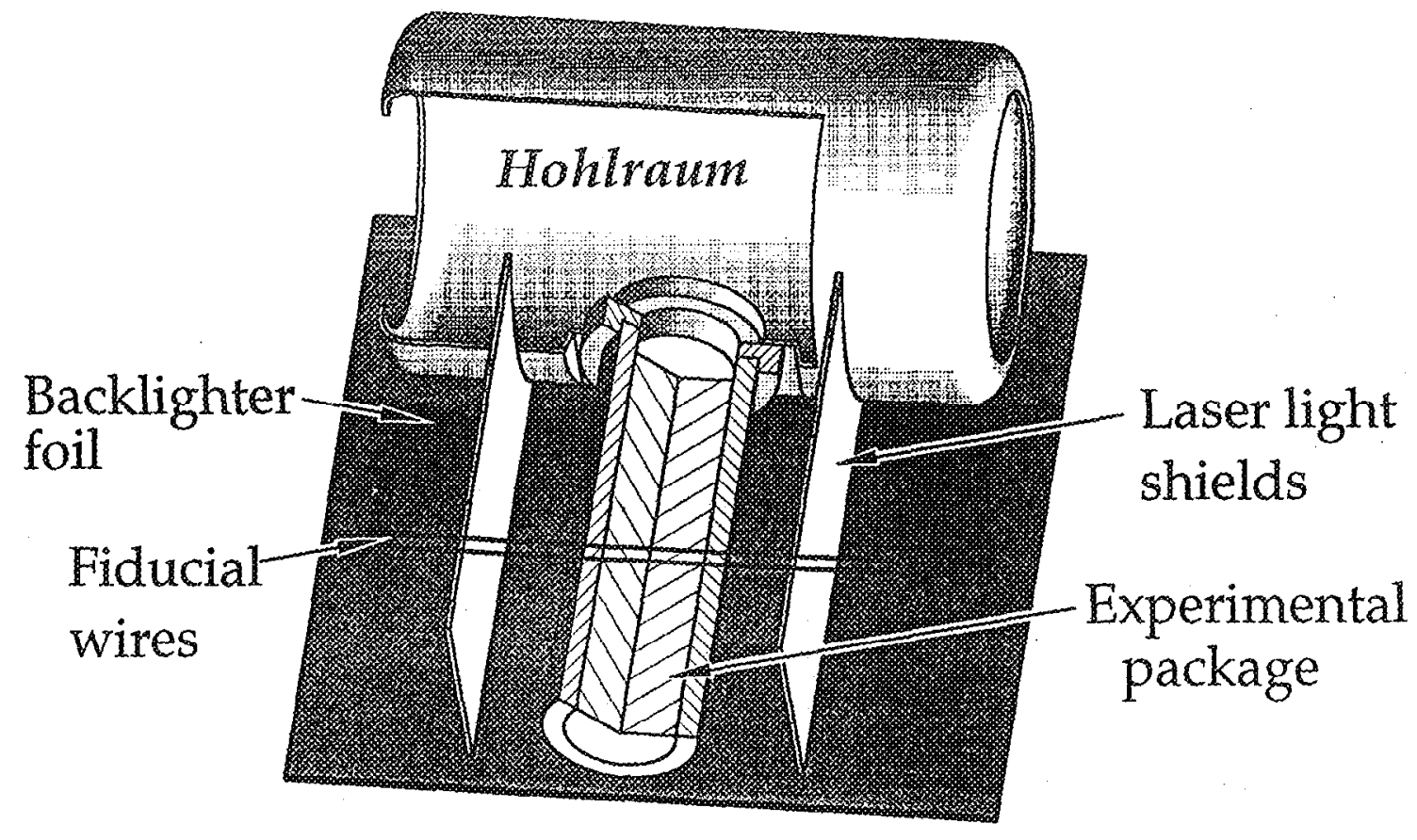

C.hlo routh 


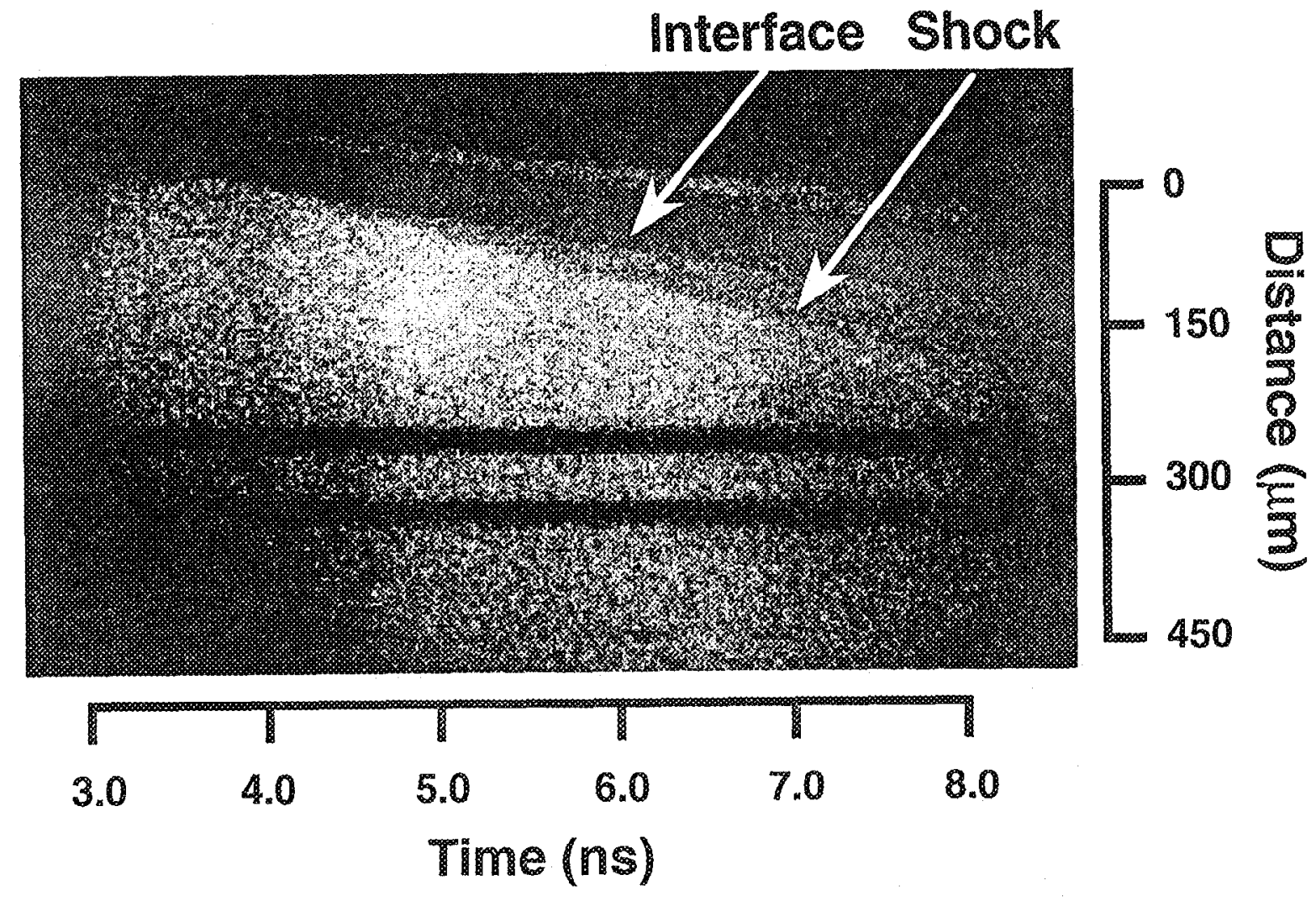




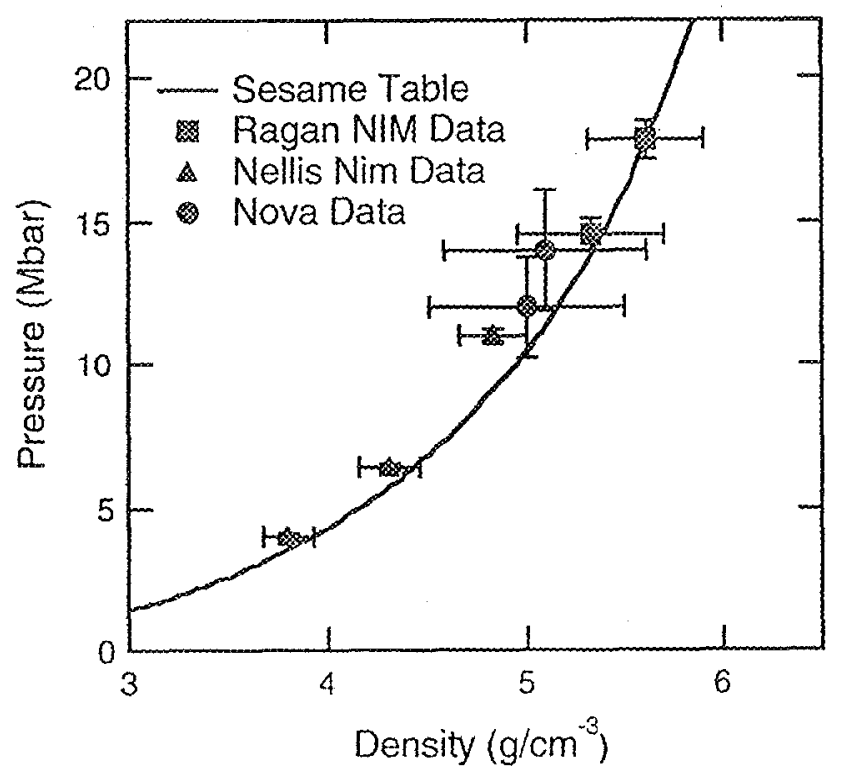




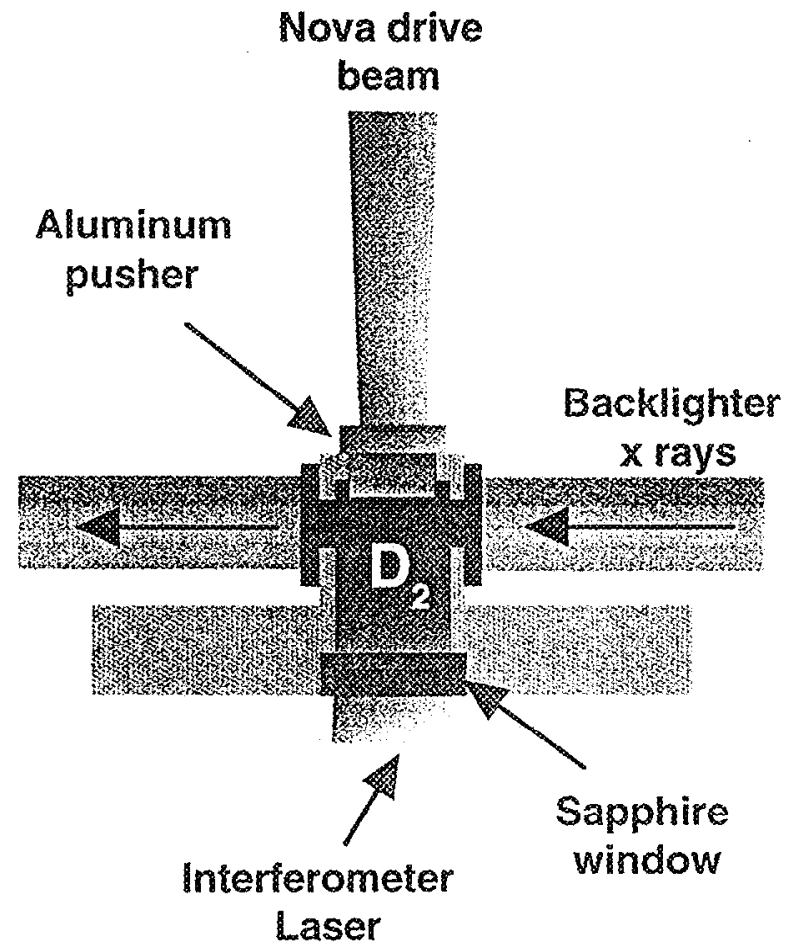

Cauble Fig $5 a$ 


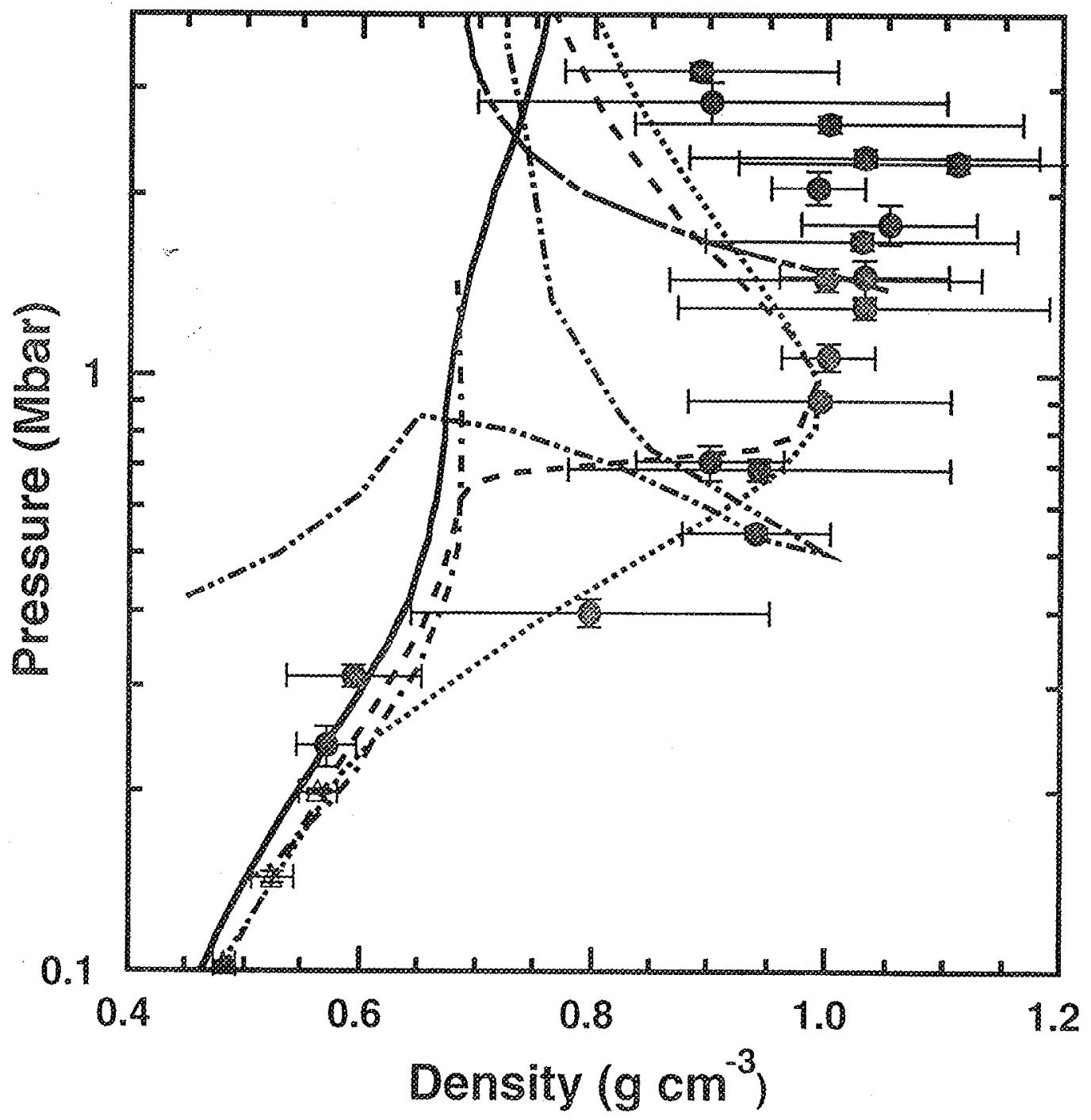

Cauble Fig $5 b$ 

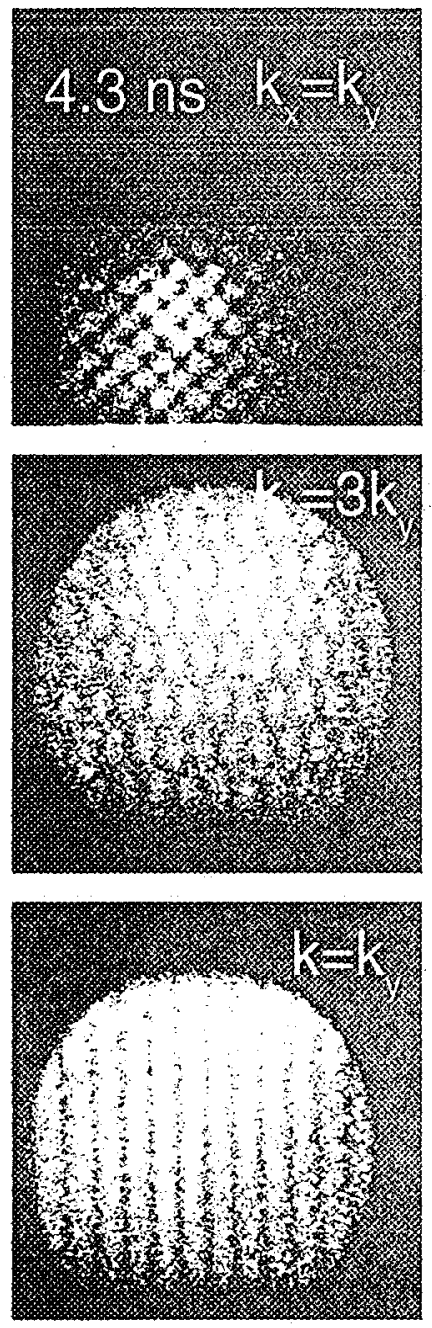

Cauble Fig $6 a$ 


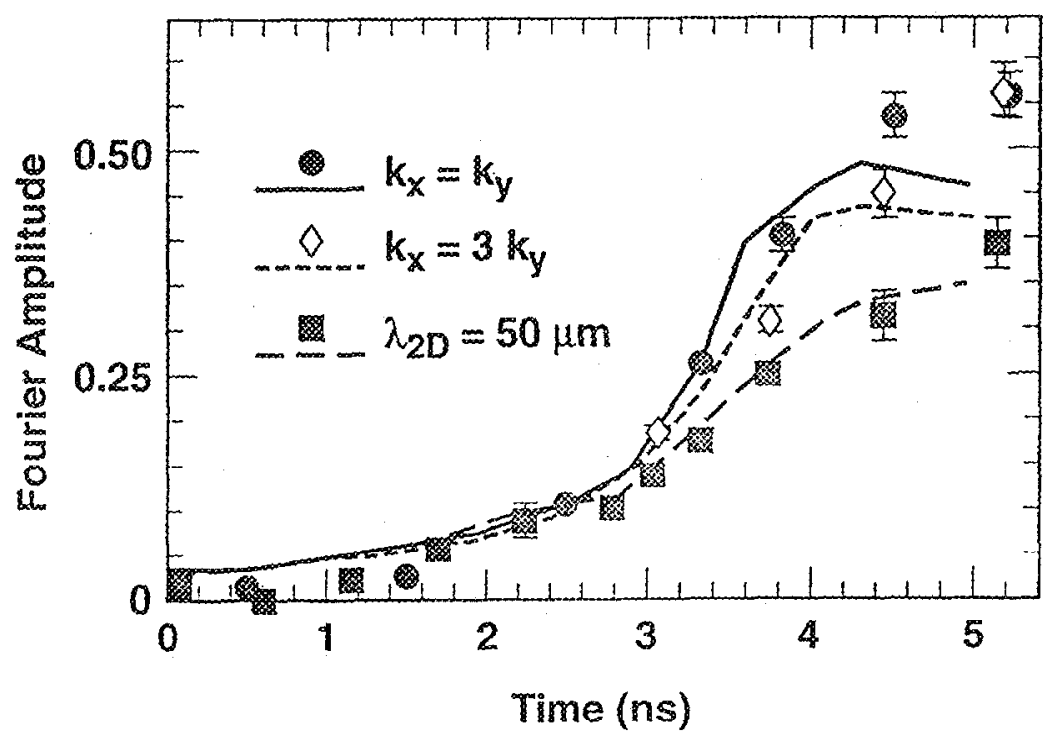

Cauble Fig $6 b$ 